Dermatology 2008;217:281-283

DOI: $\underline{10.1159 / 000150602}$

\section{Quantitative HLA-G Expression in Metastasising and Non-Metastasising Primary Thin Cutaneous Melanomas}

\author{
Michael Bezuhly a , Andrew Howlett ${ }^{\mathrm{e}}$, Patricia Colp ${ }^{\mathrm{b}}$, \\ David M. Conrad ${ }^{c}$, Noreen Walsh ${ }^{\mathrm{b}}$, Geoffrey Rowden ${ }^{\mathrm{b}}$, \\ Steven F. Morris ${ }^{\mathrm{a}}$, Richard G. Langley ${ }^{\mathrm{d}}$ \\ ${ }^{a}$ Division of Plastic Surgery, Department of Surgery, \\ Departments of ${ }^{b}$ Pathology and ${ }^{\mathrm{C}}$ Microbiology and \\ Immunology, ${ }^{\mathrm{d}}$ Division of Dermatology, Department of \\ Medicine, Dalhousie University, Halifax, N.S., and ${ }^{\mathrm{F}}$ Faculty of \\ Medicine, University of Toronto, Toronto, Ont., Canada
}

\section{Key Words}

Human lymphocyte antigen $\mathrm{G} \cdot$ Melanoma $\cdot$ Metastasis

Progression to metastatic disease in patients with thin melanomas is a relatively rare occurrence. Given the global trend towards the diagnosis of thinner lesions, however, the ability to identify lesions that will metastasise is invaluable. Considerable attention has been paid to the non-classical human lymphocyte antigen (HLA) class 1 molecule HLA-G as a candidate marker of metastatic potential. Despite the interest in HLA-G, its role in clinical prognostication remains controversial. To this end, we conducted a retrospective, matched case-control study to examine the quantitative expression of HLA-G in thin primary cutaneous melanomas that had or had not gone on to metastasise at a minimum follow-up of 7 years.

\section{Methods}

Institutional ethics committee approval was obtained. Cases of metastasising thin cutaneous melanoma were identified through the Nova Scotia Cancer Registry (NSCR) as well as a systematic review of all Dalhousie-University-affiliated hospital pathology databases. The NSCR is the central comprehensive database for registered cancers, collecting data from sources including pathology reports, registry report forms, cancer care referrals and death certificates. All cases of primary thin (Breslow thickness $\leq 1.00$ $\mathrm{mm}$ ) metastasising and non-metastasising melanoma between 1995 and 2003 were identified through the NSCR and confirmed by patient chart review. Pathological specimens were reviewed by an independent, blinded dermatopathologist (N.W.). Specimens of adequate quality were submitted for immunohistochemical assessment. For each metastasising case, 2 non-metastasising cases with a minimum of 7 years' follow-up were identified through the NSCR and matched for gender, melanoma subtype (as recorded on the original pathology report), Breslow thickness, physical site (head/neck, torso, lower limb, upper limb) and age. Individual patient data sets were coded to maintain confidentiality.

Specimens were co-processed for standardisation. Specimens were deparaffinised using xylene, rehydrated through a graded series of ethanol and rinsed in phosphate-buffered saline. Sections were submitted to antigen retrieval at high temperature and pressure in $10 \mathrm{~mm}$ sodium citrate buffer ( $\mathrm{pH}$ 6.0). Endogenous peroxidase was neutralised by incubation in $2 \%$ hydrogen peroxide. Nonspecific antibody binding was prevented through Dako protein block solution (Dako Canada Inc., Mississauga, Canada). Sections were incubated with primary monoclonal antibody against native and denatured HLA-G heavy chain $(4 \mathrm{H} 84, \mathrm{IgG1}$; gift of M. McMaster, University of California, San Francisco, Calif., USA). Omission of the primary antibody served as a negative control. Human placenta served as positive tissue control. Primary antibodies were revealed using the DakoCytomation streptavidin-biotin universal detection system according to the manufacturer's instructions (Dako Canada Inc.). Sections were counterstained with Mayer's haematoxylin and mounted. Digital images were acquired using the Nikon Coolpix 990 digital camera on a Nikon Eclipse 660 microscope (Nikon Canada, Mississauga, Canada). Quantification of staining intensity was carried out using the Imaging Processing and Analysis module of the SimplePCI Imaging System (Compix Inc., Cranberry Township, Pa., USA). Briefly, for each section all positively and negatively staining areas were delineated and the absolute light transmission of the positively staining areas measured at $\times 20$ magnification. All positive areas representing melanocytes were included, with care being taken to avoid inclusion of lymphocytes and other inflammatory cells. The transmission of each defined positive area was divided by the total area of positive staining to obtain an average transmission for each section. Average transmission is inversely related to staining intensity; 4-6 sections were processed for each lesion and the values averaged. All measurements were taken by 2 independent, blinded observers.

Results

Nine hundred cases of primary thin (Breslow thickness $\leq 1.00$ $\mathrm{mm}$ ) melanoma were recorded in the Nova Scotia Cancer Registry from 1995 to 2003. Of these cases, 20 were recorded with at least 1 episode of metastatic development. Upon patient chart review, 5 cases were excluded: 2 were out-of-province residents and 3 involved metastases from another primary cancer. Of the 13 remaining cases, 6 had specimens of adequate quality for further immunohistochemical assessment. Two additional specimens were identified through the Saint John Regional Hospital pathology database, evaluated as above, and included in the study. From the NSCR, 16 matched thin melanomas were identified that failed to show any evidence of metastasis over a minimum of 7 years' follow-up (table 1).

\section{KARGER}

(C) 2008 S. Karger AG, Basel

Fax +41 613061234

E-Mail karger@karger.ch

www.karger.com 

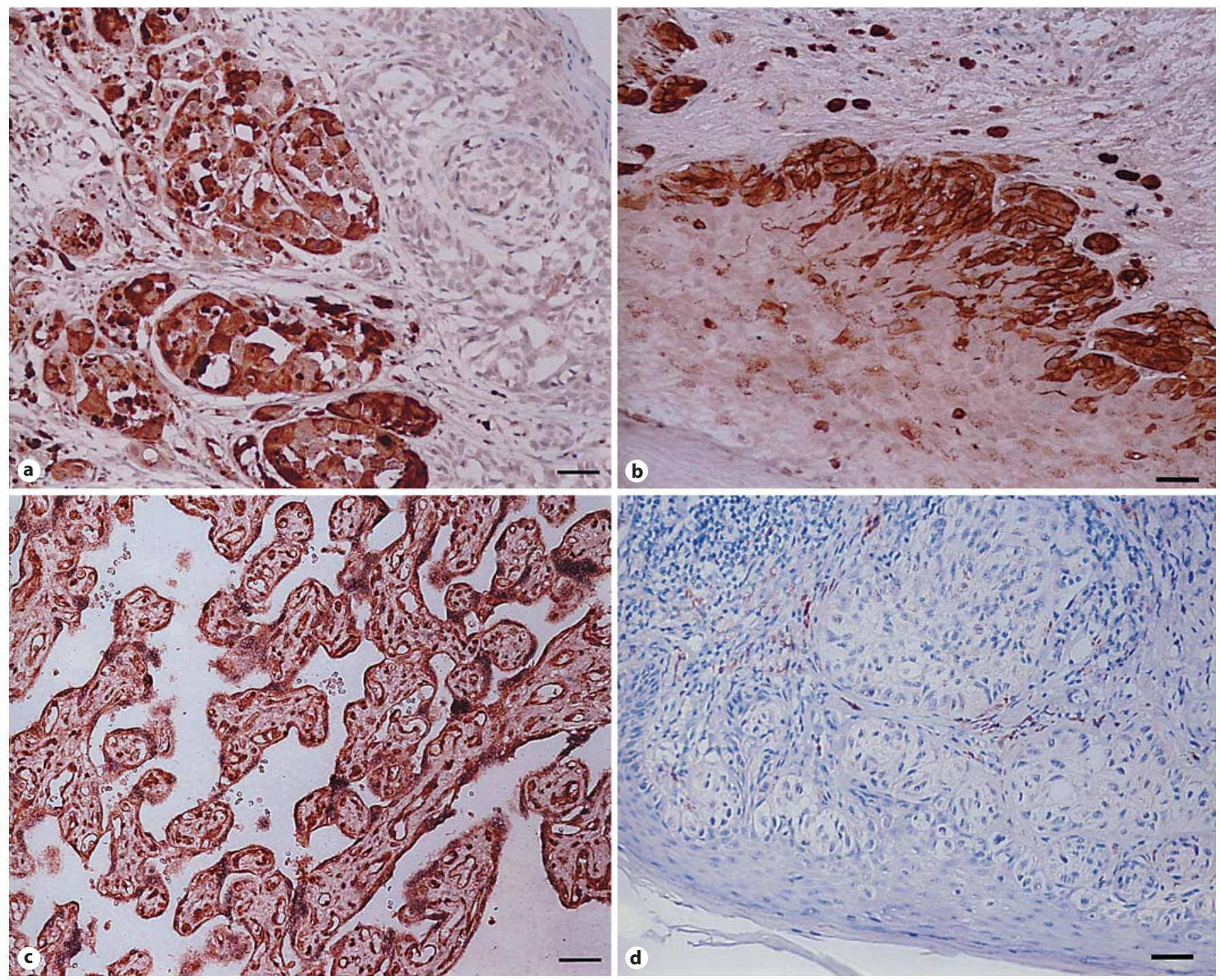

Fig. 1. HLA-G expression in thin (Breslow thickness $\leq 1.00 \mathrm{~mm}$ ) primary melanomas. Immunohistochemical staining was performed using the $4 \mathrm{H} 84$ monoclonal antibody against the HLA-G heavy chain. Bars $=40 \mu \mathrm{m}$. a Metastasising thin melanoma. b Non-metastasising thin melanoma. c Placenta (positive control). d Omission of primary antibody (negative control).

All lesions demonstrated areas of positive HLA-G staining. For metastasising thin melanomas, the average ratio of positively to negatively staining areas was $0.48 \pm 0.51$, while for non-metastasising thin melanomas it was $0.34 \pm 0.50(\mathrm{p}=0.55)$.

A Wilcoxon signed rank sum test revealed that no statistically significant difference existed between the metastasising (average transmission 0.0161) and non-metastasising (average transmission 0.0157 ) thin melanoma specimens with regards to HLA-G staining intensity $(\mathrm{p}=0.95)$. Absolute transmission values of 2.13 and 0.0132 were obtained for the negative and positive controls, respectively (fig. 1). On qualitative assessment, 6/8 metastasising cases and 9/16 non-metastasising cases demonstrated some de- gree of associated inflammatory cell infiltration. Histological regression, growth phase and mitotic activity were not assessed.

\section{Discussion}

Expressed primarily on human placental trophoblasts, the non-classical HLA class 1 molecule HLA-G has been shown to play a role in maintaining an immuno-privileged environment at the materno-fetal interface [1]. In 1998, Paul et al. [2] described HLA-G expression by melanoma cells, implicating it in cancer immune evasion. HLA-G protein expression in surgically removed benign and malignant lesions of melanocytic origin has been examined by several groups of investigators, with conflict- 
Table 1. Characteristics of metastasising and non-metastasising cohorts of patients with thin malignant melanoma

\begin{tabular}{lll}
\hline & $\begin{array}{l}\text { Patients with metastatic } \\
\text { disease }(\mathrm{n}=8)\end{array}$ & $\begin{array}{l}\text { Patients without metastatic } \\
\text { disease }(\mathrm{n}=16)\end{array}$ \\
\hline Mean \pm SD age at diagnosis, years & $56.0 \pm 13.8$ & $56.9 \pm 10.8$ \\
Male:female & $1: 1$ & $1: 1$ \\
Mean \pm SD survival/disease-free follow-up, years ${ }^{1}$ & $2.9 \pm 0.9$ & $8.3 \pm 0.8$ \\
Anatomic sites & head/neck 2, torso 3, & head/neck 5, torso 7, \\
& lower limb 2 & $0.66 \pm 0.14$ \\
Mean \pm SD Breslow thickness, mm & $0.72 \pm 0.17$ & SSM 11, NM 4, ALM 1 \\
Melanoma subtypes & SSM 6, NM 2 & $0 / 16$ \\
Ulceration & $0 / 8$ & $9 / 16$ \\
Inflammatory cell infiltrate & $6 / 8$ & \\
\hline
\end{tabular}

SSM = Superficial spreading melanoma; NM = nodular melanoma; ALM = acrolentiginous melanoma.

${ }^{1}$ For metastatic cases, time from primary diagnosis to death; for non-metastatic cases, time represents disease-free follow-up to present.

ing results [3-7]: 2 of the 6 groups have shown HLA-G protein expression in about $30 \%$ of melanoma lesions analysed, while the remainder of studies has shown no expression [8]. Much of the controversy regarding HLA-G and the clinical progression of melanoma we feel stems from the qualitative manner in which its expression has been assessed to date. For the purposes of our study, we focused on thin (Breslow thickness $\leq 1.00 \mathrm{~mm}$ ) melanomas. These lesions have a favourable prognosis, with a 10 -year metastasis rate of between 1.8 and 8.5\% [9]. We postulated that a relative increase in the level of surface HLA-G, not simply its presence, allows certain thin melanomas to metastasise. Our quantitative study appears to bear out the earlier findings of Ibrahim et al. [7]. In their study of formalin-fixed benign melanocytic lesions and primary and metastatic melanoma lesions, they showed that HLA-G expression in the primary melanomas failed to correlate with survival, time to recurrence or Breslow thickness. Although this study was informative, the omission of appropriate staining controls undermined its findings. In contrast, the only other published study to examine a series of primary melanomas showed no HLA-G expression [4]. Although we cannot definitively conclude that HLA-G is inutile as a metastatic marker given the low number of cases in our study, our quantitative findings do strengthen the argument that HLA-G is not particularly useful in this regard. Larger quantitative studies would be helpful in resolving the ongoing debate over the role of HLA-G in metastatic melanoma.

\section{Acknowledgements}

The study received financial support from the Capital District Health Authority Research Fund. A special thanks to A.M.E. O'Brien of the Saint John Regional Hospital Pathology Department, Saint John, for providing additional specimens. We would also like to thank T. Olynych of the Faculty of Medicine, Dalhousie University, for his technical support, and M. McMaster of University of California, San Francisco, for the kind gift of the antibody.

\section{References}

$\checkmark 1$ Carosella ED, Rouas-Freiss N, Paul P, Dausset J: HLA-G: a tolerance molecule from the major histocompatibility complex. Immunol Today 1999;20:60-62.

2 Paul P, Rouas-Freiss N, Khalil-Daher I, Moreau P, et al: HLA-G expression in melanoma: a way for tumor cells to escape from immunosurveillance. Proc Natl Acad Sci USA 1998;95:4510-4515.

3 Real LM, Cabrera T, Collado A, Jimenez P, Garcia A, Ruiz-Cabello F, et al: Expression of HLA-G in human tumors is not a frequent event. Int J Cancer 1999;81:512-518.

4 Frumento G, Franchello S, Palmisano GL, Nicotra MR, et al: Melanomas and melanoma cell lines do not express HLA-G, and the expression cannot be induced by gamma-IFN treatment. Tissue Antigens 2000;56: 30-37.

5 Paul P, Cabestre FA, Le Gal FA, Khalil-Daher I, et al: Heterogeneity of HLA-G gene transcription and protein expression in malignant melanoma biopsies. Cancer Res 1999;59:1954-1960.

6 Wagner SN, Rebmann V, Willers CP, Grosse-Wilde H, et al: Expression analysis of classic and non-classic HLA molecules before interferon alfa-2b treatment of melanoma. Lancet 2000;356:220-221.

7 Ibrahim EC, Aractingi S, Allory Y, Borrini F, et al: Analysis of HLA antigen expression in benign and malignant melanocytic lesions reveals that up-regulation of HLA-G expression correlates with malignant transformation, high inflammatory infiltration and HLA-A1 genotype. Int J Cancer 2004;108:243-250.

8 Chang CC, Ferrone S: HLA-G in melanoma: can the current controversies be solved? Semin Cancer Biol 2003;13:361-369.

9 Schmid-Wendtner M-H, Baumert J, Eberle J, Plewig G, et al: Disease progression in patients with thin cutaneous melanomas (tumour thickness $\leq 0.75 \mathrm{~mm}$ ): clinical and epidemiological data from the Tumour Centre Munich 1977-1998. Br J Dermatol 2003;149:788-793.

Richard Langley, MD, FRCPC

4195 Dickson Building, Queen Elizabeth II Health Sciences Centre 5820 University Avenue, Dalhousie University

Halifax, Nova Scotia B3H 1 V7 (Canada)

Tel. +1 902425 8038, Fax +1902421 1580

E-Mail richard.langley@dal.ca 